\title{
25 Research Square \\ Rapid and Reliable Analysis of Kojic Acid in Fungal Agar
}

Masayo Kushiro ( $\nabla$ kushirom@affrc.go.jp )

National Agriculture and Food Research Organization https://orcid.org/0000-0002-8876-0316

Koji Aoyama

National Agriculture and Food Research Organization

Marin Kishimoto

National Agriculture and Food Research Organization

Yasujiro Morimitsu

Ochanomizu Joshi Daigaku

Tomohiro Furukawa

National Agriculture and Food Research Organization

\section{Short Report}

Keywords: Aspergillus, secondary metabolite, kojic acid, HPLC, spectrometry

Posted Date: November 8th, 2021

DOI: https://doi.org/10.21203/rs.3.rs-1047138/v1

License: (c) (1) This work is licensed under a Creative Commons Attribution 4.0 International License.

Read Full License 


\section{Abstract}

A rapid HPLC-UV method was developed for the quantitative analysis of kojic acid contained in fungal agar media. The methanol extract from fungal agar media was diluted with acetic acid aqueous solution and injected into HPLC. The separation was achieved on a C18 column within 10 minutes in an isocratic mode. The mobile phase was composed of water, methanol, and acetic acid. Spike and recovery tests were done using three representative agar media, and accuracy, precision and limit of detection were preferable. The developed method was applicable for the analysis of kojic acid in two Aspergillus strains practically, showing the usability of this method to evaluate the fungal ability to produce a beneficial secondary metabolite, kojic acid.

\section{Introduction}

Aspergillus section Flavi is a producer of vast and various kinds of secondary metabolites [1]. Among them, kojic acid (5-hydroxy-2-(hydroxymethyl)-4H-pyran-4-one) is a naturally occurring organic acid with the molecular formula of $\mathrm{C}_{6} \mathrm{H}_{6} \mathrm{O}_{4}$, discovered in 1907 [2]. It acts as an ultra-violet protector by suppressing hyper-pigmentation in animal skins restraining the formation of melanin (skin pigment) through the inhibition of tyrosinase, the key enzyme involved in melanogenesis pathway [3].

Because of medicinal and cosmetic application of kojic acid in the form of external agent such as ointment, many attempts to increase the contents of kojic acid in some species of genus Aspergillus have been reported [4,5]. Japanese researchers had contributed to the development of analytical method for kojic acid using GC/MS and HPLC [6,7]. The most common analytical method for kojic acid in cosmetics is HPLC with UV detection. Recently, Wang et al. reported an HPLC-UV method for simultaneous determination of nine whitening agents including kojic acid within $30 \mathrm{~min}$ [8]. Furthermore, the possibility of misassignment between kojic acid and its isomer; flufuran was reported very recently [9]. Since there are an emerging number of new fungal species and continuous efforts to find competent strains to produce kojic acid, more rapid and reliable analytical method for kojic acid from fungal media is expected.

Currently, however, there is no verified analytical method for kojic acid in fungal media. TLC is often used as a qualitative and semi-quantitative method because of the ease of handling and short analytical time [4]. Here we report the development of a rapid and reliable HPLC-UV method for kojic acid in fungal agar media with simple extraction and dilution. The method was quantitatively verified with the parameters of accuracy, precision and limit of detection (LOD), and qualitatively verified with UV and MS spectrometry. The developed method was practically usable for the quantification of kojic acid among two Aspergillus isolates.

\section{Materials And Methods}


An Aspergillus isolate (MAFF 111759, A. parasiticus) obtained from NARO Genebank (Tsukuba, Japan) and another isolate JUL1 ( $A$. flavus) isolated from a field soil in Tsukuba, Japan, were used [10]. For the growth of each fungus, YES ( $2 \%$ yeast extract, $10 \%$ sucrose, $0.1 \%$ sodium deoxycholate, $0.01 \%$ chloramphenicol, $2 \%$ agar), GY2-0.5 agar medium ( $2 \%$ glucose, $0.5 \%$ yeast extract, $2 \%$ agar), and GY $5-0.5$ agar medium (5\% glucose, $0.5 \%$ yeast extract, $2 \%$ agar) were used $[11,12]$. Two microliter of spore suspension solution of each strain $\left(5-50 \times 10^{6}\right.$ spores $\left./ \mathrm{mL}\right)$ was loaded on the above agar media in triplicate, and kept in an incubation room for a week at $25^{\circ} \mathrm{C}$.

Kojic acid was purchased from Tokyo Chemical Industry (Tokyo, Japan). All other reagents were of HPLC or analytical grade. Stock solution of kojic acid $(40,000 \mathrm{mg} / \mathrm{mL})$ was prepared in $0.1 \%$ acetic acid and methanol $(9: 1, \mathrm{v} / \mathrm{v})$ and diluted to concentrations of $5,20,50,100,200$, and $500 \mathrm{mg} / \mathrm{mL}$ in $0.1 \%$ acetic acid and methanol $(9: 1, v / v)$ for a calibration curve.

Analysis throughout the study was done in triplicate $(n=3)$. Approximately one gram of agar debris with fungal colony was scratched and extracted with five times volume of methanol $(5 \mathrm{~mL}$ per $1 \mathrm{~g})$ in $15 \mathrm{~mL}$ centrifuge tubes by $1 \mathrm{~min}$ of vortex and $3 \mathrm{~min}$ of sonication. After centrifugation (3,000 rpm, $10 \mathrm{~min}), 0.1$ $\mathrm{mL}$ of supernatant was transferred to a $1.5 \mathrm{~mL}$ microtube and $0.9 \mathrm{~mL}$ of $0.1 \%$ acetic acid was added. The diluted sample was centrifuged (10,000 rpm, $5 \mathrm{~min})$ and the supernatant was transferred to an HPLC vial to serve for HPLC-UV analysis. An LC1100 series HPLC system connected to a photodiode array detector (Agilent Technologies, Santa Clara, CA) was used, and data were acquired and integrated using ChemStation ver. C.01.07 Software (Agilent). An isocratic HPLC condition was adopted at a mobile phase of $0.1 \%$ acetic acid and methanol $(9: 1, \mathrm{v} / \mathrm{v}$ ) and a flow rate of $1 \mathrm{~mL} / \mathrm{min}$. An L-column ODS (250 by 4.6 $\mathrm{mm}$ inside diameter, $5 \mathrm{~mm}$ spherical particle size; CERI, Tokyo, Japan) was used at $40^{\circ} \mathrm{C}$, and the injection volume was $10 \mathrm{~mL}$. The detector was set at a wavelength of $270 \mathrm{~nm}$. When the content was outside of the calibration curve, the sample was diluted as appropriate. The limit of detection (LOD) was $1 \mathrm{mg} / \mathrm{kg}$ as a concentration with a signal-to-noise ratio of 3 or more and distinguishable from adjacent peaks. Spike and recovery tests were performed at levels of 100 and 2,000 mg/kg. First, each $1 \mathrm{~g}$ of blank agar debris scratched from media was put into a $15 \mathrm{~mL}$ centrifuge tube. Then, $0.05 \mathrm{~mL}$ of standard kojic acid solution $(2,000$ or $40,000 \mathrm{mg} / \mathrm{mL}$ in $0.1 \%$ acetic acid and methanol $(9: 1, \mathrm{v} / \mathrm{v}))$ was added to the agar debris, and $5 \mathrm{~mL}$ of methanol was added after 5 minutes. The procedure after extraction was the same as described above.

UV spectra data of the standard of kojic acid and samples were also obtained in the same detectorat the absorbance wavelength range from 200 to $400 \mathrm{~nm}$ (Agilent). Further identification of kojic acid was conducted with a high-resolution (HR)-MS (Orbitrap MS "Exactive") (Thermo Fisher Scientific, Waltham, MA) connected with LC using $0.1 \%$ formic acid aqueous solution (solvent $A$ ) and acetonitrile (solvent $B$ ) [13]. The gradient condition was 5\% B (0-3.0 min), 5-90\% B (3.0-18.0 min), 90\% B (18.0-22.0 min), 90-5\% B (22.0-22.1 $\mathrm{min})$, and $5 \% \mathrm{~B}(22.1-29.0 \mathrm{~min})$. The flow rate was set to $0.45 \mathrm{~mL} / \mathrm{min}$ and the column temperature was $40^{\circ} \mathrm{C}$ equipped with Capcell Pak UG120 C18 column $(150 \mathrm{~mm}$ by $4.6 \mathrm{~mm}$ inside diameter, $3 \mu \mathrm{m}$ spherical particle size; Osaka Soda Co., Ltd., Osaka, Japan). The injection volume was 10 $\mu \mathrm{L}$. MS was equipped with a heated electrospray ionization (HESI) probe, and the parameters are as 
listed: heater temperature, $120^{\circ} \mathrm{C}$; sheath gas, 30 ; auxiliary gas, 5 ; sweep gas, 0 ; spray voltage, $4.0 \mathrm{kV}$; Capillary temperature, $250^{\circ} \mathrm{C}$. The system was operated in a positive mode to detect the ion of $[\mathrm{M}+\mathrm{H}]^{+}$ $\left(\mathrm{C}_{6} \mathrm{H}_{7} \mathrm{O}_{4}\right)$. A full scan range was set at 80 to $500(\mathrm{~m} / \mathrm{z})$, and the resolution was set at $100,000(\mathrm{at} \mathrm{m} / \mathrm{z}$ 200).

\section{Results And Discussion}

To verify the analytical method for kojic acid quantitatively, spike and recovery tests were conducted using YES, GY2-0.5 and GY5-0.5 agar medium. According to the survey by Kimura et al., spike level to food matrices (organic matrices) was set at $100 \mathrm{mg} / \mathrm{kg}$ [14]. In this study, we set the spiking concentrations at $100 \mathrm{mg} / \mathrm{kg}$ and additively $2000 \mathrm{mg} / \mathrm{kg}$ for higher level of production in fungal agar media. Selectivity and resolution of kojic acid in chromatogram were acceptable in YES and GY agar matrices (Fig. 1). Recovery values were calculated as the average of triplicate data with a relative standard deviation (RSDr) shown in Table 1. The recovery percentages ranged from 74.0 to $110 \%$, the range generally accepted as an in-house validation of an analytical method. The RSDr values ranged from 3.1 and $16 \%$, being unstable and over $5 \%$, the value generally accepted as an in-house validation of an analytical method at this level [15], but we consider that it will not matter for practical analyses. The recovery rate is fair, and therefore, the analytical method described here should be applicable for the practical analysis of kojic acid in agar media.

The LODs of previously reported two HPLC-UV methods for kojic acid were $5 \mathrm{mg} / \mathrm{kg}$ and $1 \mathrm{mg} / \mathrm{kg}$ for cosmetics and food, respectively [8,14]. In this study, the LOD calculated was $1 \mathrm{mg} / \mathrm{kg}$ in agar matrices. The LOD value is comparative or lower than those reported previously. In this study, we did not adopt buffer containing phosphate as a mobile phase [14]. Instead, we adopted a mobile phase consisted of water, methanol, and acetic acid [8]. This method does not require $\mathrm{pH}$ adjustmentbecause of simple constitution of mobile phase with short analytical time within ten minutes.

Two Aspergillus isolates MAFF 111759 (an A. parasiticus isolate deposited at Genebank) and JUL1 (an A. flavus isolate from a field soil) were served to detect kojic acid by the HPLC-UV method described above. Qualitative confirmation of kojic acid production was conducted by UV spectrometry and HR-MS. Fig. 2(A) and (B) represents contour plots with time (X-axis) and wavelength ( $\mathrm{Y}$-axis) of standard kojic acid (50 mg/mL) (A) and the extracted sample (GY5-0.5 agar grown JUL1 isolate) (B). Fig. 2(C) and (D) represents UV-absorption spectra at 5.1 min peaks of standard kojic acid (C) and the extracted sample (GY5-0.5 agar grown JUL1 isolate) (D). The contour plots ((A) and (B)) showed very high similarity and spectra showed identity $((C)$ and $(D))$, between the standard and the extracted sample.

Using the same standard and the extracted sample, HESI(+)-HR-MS analysis was conducted. The HR-MS analysis depicted the mass spectrum at LC retention time of $7.29 \mathrm{~min}$ for standard kojic acid; accordingly, $\mathrm{m} / z 143.0338[\mathrm{M}+\mathrm{H}]^{+}$(calculated for $\mathrm{C}_{6} \mathrm{H}_{7} \mathrm{O}_{4}{ }^{+}, 143.0339, \Delta \mathrm{ppm}=0.7149$ ) was obtained. On the other hand, $m / z 143.0337[\mathrm{M}+\mathrm{H}]^{+}$(calculated for $\mathrm{C}_{6} \mathrm{H}_{7} \mathrm{O}_{4}{ }^{+}, 143.0339, \Delta \mathrm{ppm}=1.2483$ ) was obtained from the mass spectrum at LC retention time of $7.23 \mathrm{~min}$ for the extracted sample (Fig. 3). Very recently the 
possibility of misidentification of kojic acid and flufuran is reported [9]. These two compounds are isomers and indistinguishable by molecular weight, but they can be distinguished from each other by UV spectra and peak purity check by 2D display to exclude any contribution of other compounds. From the combined spectrometry results of Fig. 2 and Fig. 3, the major compound found in the extracted sample is truly kojic acid. Thus, it was shown that the method developed here is practically applicable for the analysis of kojic acid in fungal agar media. This method is very simple and efficient because only a dilution of sample is needed, and cleanup procedures are not required.

Using this HPLC-UV method, the effect of glucose on kojic acid production was studied with above two fungal strains. It was shown that in both Aspergillus analyzed, higher production of kojic acid was observed at higher glucose content in GY agar (Table 2). This method will contribute to efficient screening of kojic acid producers among fungal strains deposited at Genebank and newly isolated ones, as well as to elucidate conditions for higher production of kojic acid.

\section{Declarations}

\section{Acknowledgements}

We are grateful toDr. Wichittra Arai for technical assistance. This study was partly conducted under the research project on "Regulatory research projects for food safety, animal health and plant protection (JPJ008617.18072043)" funded by the Ministry of Agriculture, Forestry and Fisheries of Japan. We thank the Advanced Analysis Center, NARO, for use of facilities.

\section{Conflict of interest}

All authors have no conflicts of interest related to this study.

\section{References}

1. Handbook of Toxic Fungal Metabolites. Richard J. Cole, Richard H. Cox, Academic Press (1981).

2. Bentley R. From miso, sake and shoyu to cosmetics: a century of science for kojic acid. Nat Prod Rep.2006;23(6):1046-62.

3. Chang T-S. An updated review of tyrosinase inhibitors. Int J Mol Sci. 2009;10(6):2440-75.

4. Chib S, Dogra A, Nandi U, Saran S. Consistent production of kojic acid from Aspergillus sojae SSC-3 isolated from rice husk. Mol Biol Rep. 2019;46(6):5995-6002.

5. Feng W, Lianf J, Wang B, Chen J. Improvement of kojic acid production in Aspergillus oryzae AR-47 mutant strain by combined mutagenesis. Bioprocess Biosyst Eng. 2019;42(5):753-61. 
6. Manabe M, Shinkai E, Goto T, Misawa Y, Tanaka K, Matsuura S. Studies on the fluoresce compound in fermented foods (Part 6), High-performance liquid chromatographic analytical system for kojic acid. Journal of Japanese Soy Sauce Research Institute (Nihon Shoyu Kenkyu-sho Zasshi, article in Japanese). 1984;10:146-50.

7. Manabe M, Shinkai E, Goto T, Tanaka K, Misawa Y. Gas-liquid chromatographic analytical system for kojic acid. Journal of Japanese Soy Sauce Research Institute (Nihon Shoyu Kenkyu-sho Zasshi, available Summary in English, article in Japanese). 1990;16:183-6.

8. Wang Y-H, Avonto C, Avula B, Wang M. Qutantitative determination of a-arbutin, b-arbutin, kojic acid, nicotinamide, hydroquinone, resorcinol, 4-methoxyphenol, 4-ethoxyphenol, and ascorbic acid from skin whitening products by HPLC-UV. Journal of AOAC International.2015;98(1):5-12.

9. DellaGreca M, De Tommaso G, Salvatore MM, Nicoletti R, Becchimanzi A, luliano M, Andolfi A. The issue of misidentification of kojic acid with flufuran in Aspergillus flavus. Molecules. 2019;24(9):1709.

10. Kishimoto M, Furukawa T, Karasawa T, Morimitsu Y, Kushiro M. Isolation of Aspergillus flavus strains from field soil by the improved DV-AM method. JSM Mycotoxins (available Abstract in English, article in Japanese). 2021;71(1):9-12.

11. Yabe K, Yushita M, Yamamoto Y, Kushiro M. Detection of aflatoxigenic fungi in imported raw nuts using the dichlorvos-ammonia (DV-AM) method. JSM Mycotoxins. 2020;70(1):7-13.

12. Kushiro M, Hatabayashi $\mathrm{H}$, Nakagawa $\mathrm{H}$, Yabe K. Detection of Aspergillus novoparasiticus from Japanese sugarcane field by the dichlorvos-ammonia (DV-AM) method with single colony AM assay. JSM Mycotoxins.2020;70(2):51-6.

13. Matsuo $Y$, Hatabayashi $H$, Nakagawa $H$. Detection of $N$-(1-deoxy-d-fructos-1-yl) fumonisin $C_{1}, C_{2}$ and $\mathrm{C}_{3}$ in corn powder by LC - Orbitrap MS. Environmental \& Analytical Toxicology. 2018;8(1):536.

14. Kimura K, Hirokado M, Yasuda K, Nishijima M Determination of kojic acid in various commercial foods by HPLC. J. Food Hygienic Soc. Japan (Shokuhin Eiseigaku Zasshi, available Abstract in English, article in Japanese). 2000;41(1):70-73.

15. AOAC International. (2005). Official method for analysis, appendix E: Laboratory quality assurance. http://www.eoma.aoac.org/app_e.pdf

\section{Tables}

Due to technical limitations, table 1-2 is only available as a download in the Supplemental Files section.

\section{Figures}


(A)

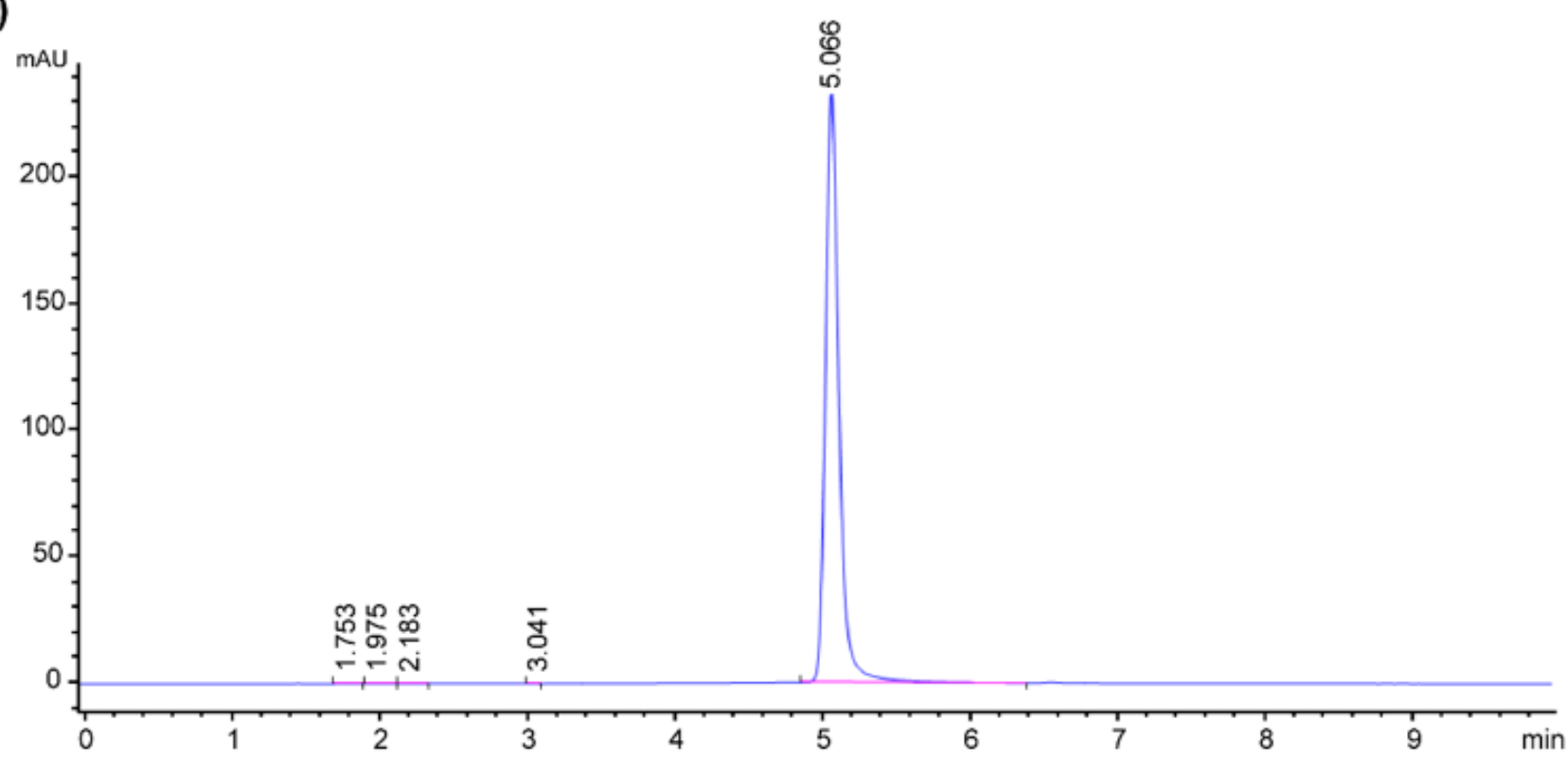

(B)

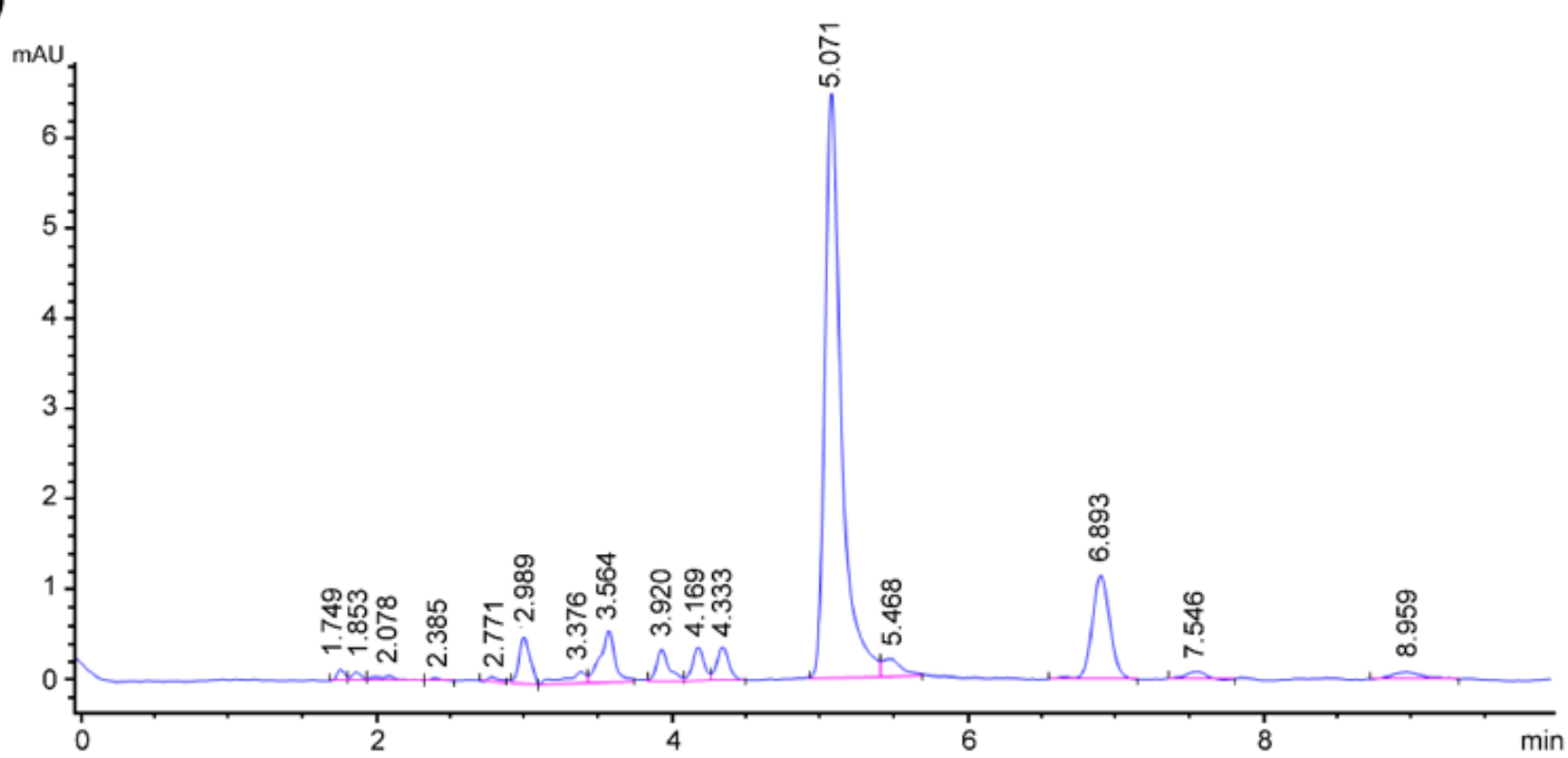

Figure 1

HPLC chromatograms of (A) standard kojic acid (50 $\mu \mathrm{g} / \mathrm{ml})$ and (B) extracted sample (GY(5-0.5) agar medium, low level spiked) 


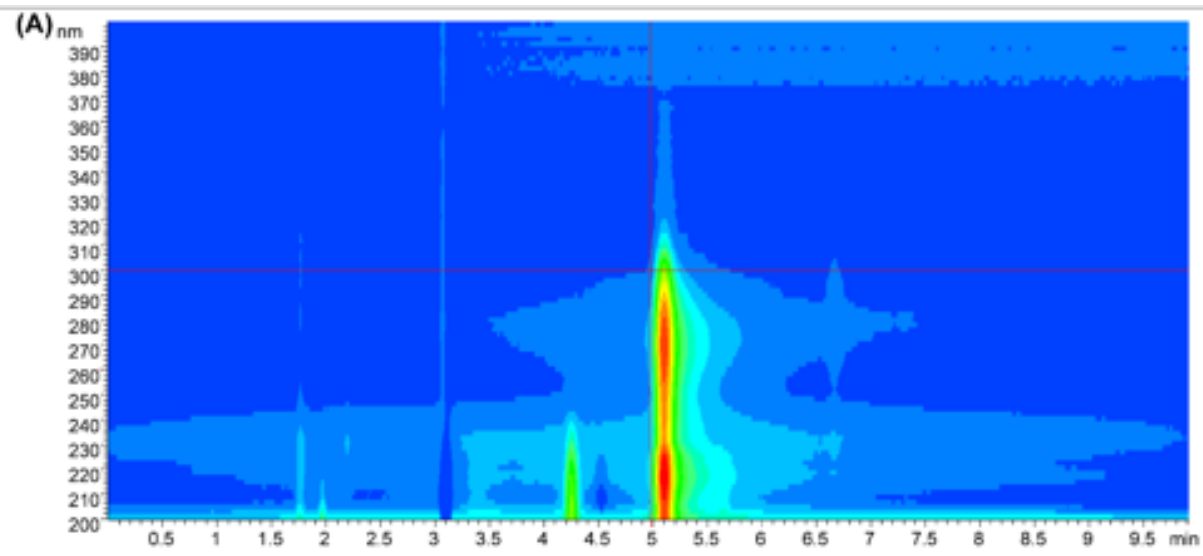

(B)

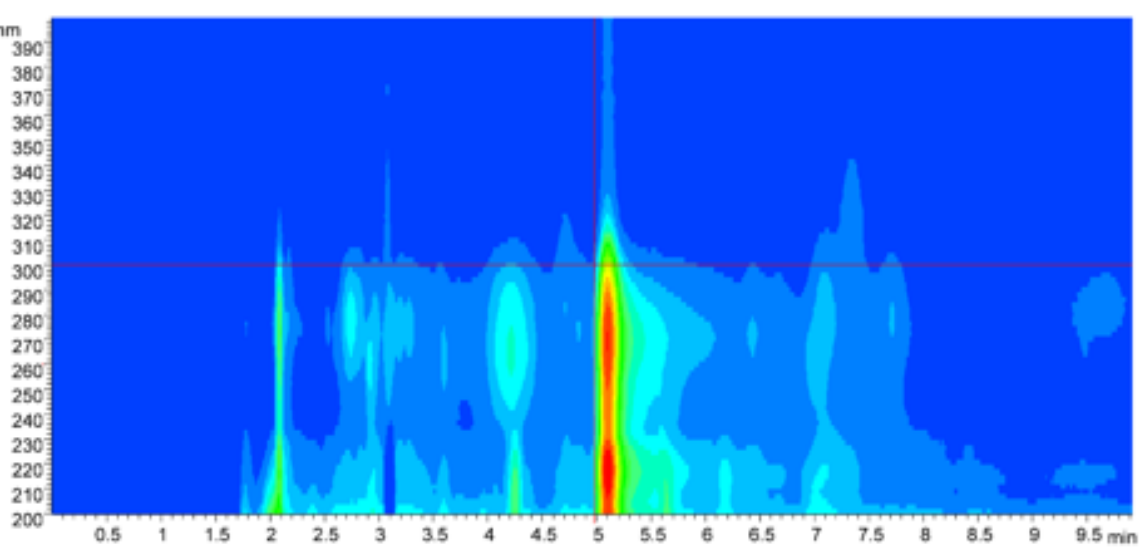

(C)

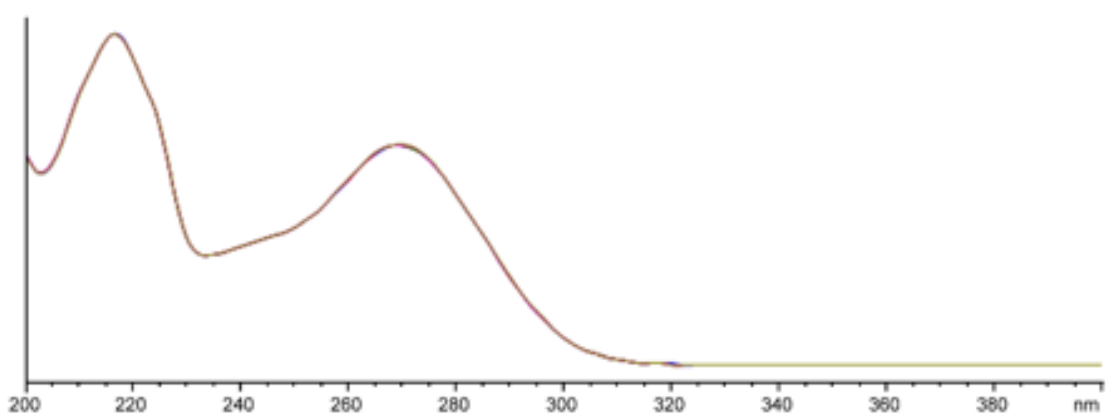

(D)

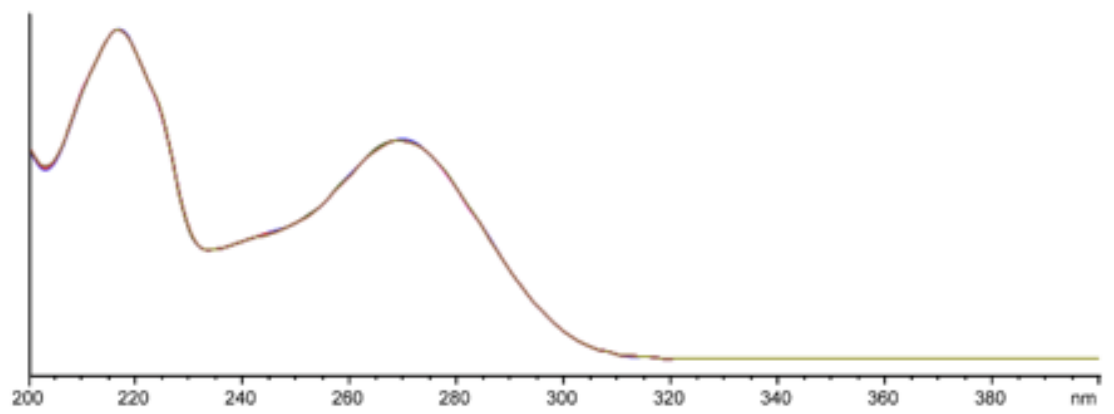

Figure 2

HPLC-UV contour plots with time (X-axis) and wavelength ( $\mathrm{Y}$-axis) of (A) standard kojic acid ( $50 \mu \mathrm{g} / \mathrm{ml})$ and (B) extracted sample (JUL1), and UV-absorption spectra at 5.1 min peaks of (C) standard kojic acid $(50 \mu \mathrm{g} / \mathrm{ml})$ and (D) extracted sample (JUL1) 

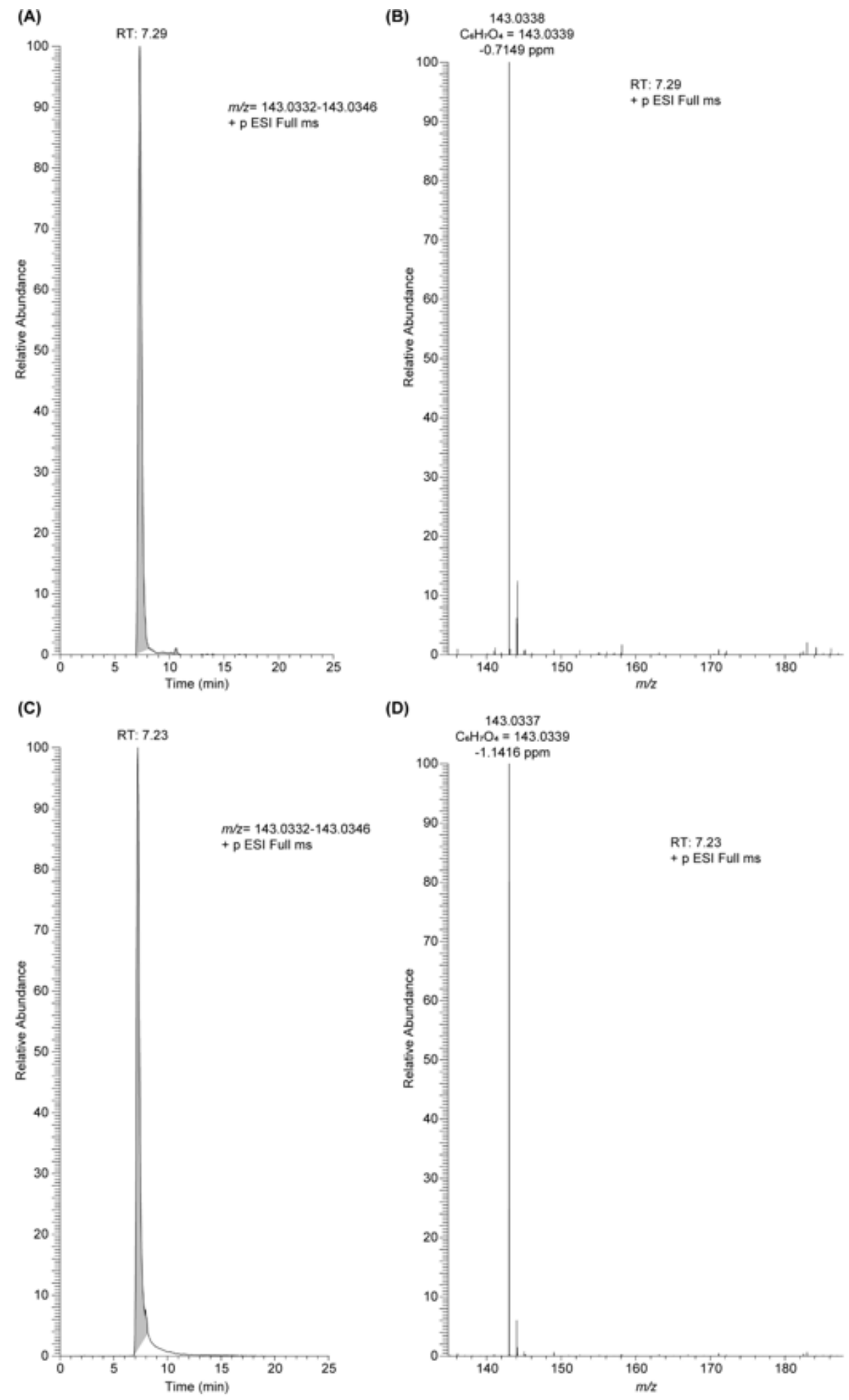

\section{Figure 3}

Selected ion monitoring (A) and full MS spectrum obtained at $7.29 \mathrm{~min}(\mathrm{~B})$ of standard kojic acid (50 $\mu \mathrm{g} / \mathrm{ml}$ ) analyzed by LC-Orbitrap MS. Selected ion monitoring (C) and full MS spectrum obtained at 7.23 min (D) of the extracted sample (JUL1) detected by LC-Orbitrap MS.

\section{Supplementary Files}


This is a list of supplementary files associated with this preprint. Click to download.

- 211102Table12.xlsx 